\title{
Re-translation versus Streaming for Simultaneous Translation
}

\author{
Naveen Arivazhagan; Colin Cherry, Wolfgang Macherey and George Foster \\ Google Research \\ \{navari, colincherry, wmach, fosterg\}@google.com
}

\begin{abstract}
There has been great progress in improving streaming machine translation, a simultaneous paradigm where the system appends to a growing hypothesis as more source content becomes available. We study a related problem in which revisions to the hypothesis beyond strictly appending words are permitted. This is suitable for applications such as live captioning an audio feed. In this setting, we compare custom streaming approaches to re-translation, a straightforward strategy where each new source token triggers a distinct translation from scratch. We find retranslation to be as good or better than stateof-the-art streaming systems, even when operating under constraints that allow very few revisions. We attribute much of this success to a previously proposed data-augmentation technique that adds prefix-pairs to the training data, which alongside wait- $k$ inference forms a strong baseline for streaming translation. We also highlight re-translation's ability to wrap arbitrarily powerful MT systems with an experiment showing large improvements from an upgrade to its base model.
\end{abstract}

\section{Introduction}

In simultaneous machine translation, the goal is to translate an incoming stream of source words with as low latency as possible. A typical application is speech translation, where we often assume the eventual output modality to also be speech. In a speech-to-speech scenario, target words must be appended to existing output with no possibility for revision. The corresponding translation task, which we refer to as streaming translation, has received considerable recent attention, generating custom approaches designed to maximize quality and minimize latency (Cho and Esipova, 2016; Gu et al.,

\footnotetext{
${ }^{*}$ Equal contributions
}

2017; Dalvi et al., 2018; Ma et al., 2019a). However, for applications where the output modality is text, such as live captioning, the prohibition against revising output is overly stringent.

The ability to revise previous partial translations makes simply re-translating each successive source prefix a viable strategy. Compared to streaming models, re-translation has the advantage of low latency, since it always attempts a translation of the complete source prefix, and high final-translation quality, since it is not restricted to preserving previous output. It has the disadvantages of higher computational cost, and a high revision rate, visible as textual instability in an online translation display. When revisions are an option, it is unclear whether one should prefer a specialized streaming model or a re-translation strategy.

In light of this, we make the following contributions: (1) We evaluate a combination of retranslation techniques that have not previously been studied together. (2) We provide the first empirical comparison of re-translation and streaming models, demonstrating that re-translation operating in a very low-revision regime can match or beat the quality-latency trade-offs of streaming models. (3) We test a 0 -revision configuration of re-translation, and show that it is surprisingly competitive, due to the effectiveness of data augmentation with prefix pairs.

\section{Related Work}

Cho and Esipova (2016) propose the first streaming techniques for NMT, using heuristic agents based on model scores, while Gu et al. (2017) extend their work with agents learned using reinforcement learning. Ma et al. (2019a) recently broke new ground by integrating their read-write agent directly into NMT training. Similar to Dalvi et al. (2018), they employ a simple agent that first reads $k$ source to- 
kens, and then proceeds to alternate between writes and reads until the source sentence has finished. This agent is easily integrated into NMT training, which allows the NMT engine to learn to anticipate occasionally-missing source context. We employ their wait- $k$ training as a baseline, and use their wait- $k$ inference to improve re-translation. Our second and strongest streaming baseline is the MILk approach of Arivazhagan et al. (2019b), who improve upon wait- $k$ training with an attention that can adapt how it will wait based on the current context. Both wait- $k$ training and MILk attention provide hyper-parameters to control their qualitylatency trade-offs: $k$ for wait- $k$, and latency weight for MILk.

Re-translation was originally investigated by Niehues et al. (2016, 2018), and more recently extended by Arivazhagan et al. (2019a), who propose a suitable evaluation framework, and use it to assess inference-time re-translation strategies for speech translation. We adopt their inference-time heuristics to stabilize re-translation, and extend them with prefix training from Niehues et al. (2018). Where they experiment on TED talks, compare only to vanilla re-translation and use proprietary NMT, we follow recent work on streaming by using WMT training and test data, and provide a novel comparison to streaming approaches.

\section{Metrics}

We adapt the evaluation framework from Arivazhagan et al. (2019a), which includes metrics for latency, stability, and quality. Where they measure latency with a temporal lag, we adopt an established token lag that does not rely on machine speed.

Our evaluation is built around a prefix translation list (PTL), which can be generated for any streaming or re-translation system. For each token in the source sentence (after merging subwords), this list stores the tokenized system output. Table 1 shows an example. We use $I$ for the final number of source tokens, and $J$ for the final number of target tokens.

\subsection{Quality}

Translation quality is measured by calculating BLEU (Papineni et al., 2002) on the final output of each PTL; that is, standard corpus-level BLEU on complete translations. Specifically, we report tokenized, cased BLEU calculated by an internal tool. We make no attempt to directly measure the quality of intermediate outputs; instead, their quality is captured indirectly through final output quality and stability.

\subsection{Latency}

Latency is the amount of time the target listener spends waiting for their translation. Most latency metrics are based on a delay vector $g$, where $g_{j}$ reports how many source tokens were read before writing the $j^{\text {th }}$ target token (Cho and Esipova, 2016). This delay is trivial to determine for streaming systems, but to address the scenario where target content can change, we introduce the notion of content delay, which is closely related to the finalization event index used to calculate time delay in Arivazhagan et al. (2019a).

We take the pessimistic view that content in flux is useless; for example, in Table 1 , the $4^{\text {th }}$ target token first appears in step 4, but only becomes useful in step 7, when it shifts from be to slow. Therefore, we calculate delay with respect to when a token finalizes. Let $o_{i, j}$ be the $j^{\text {th }}$ token of the $i^{\text {th }}$ output in a PTL; $1 \leq i \leq I$ and $1 \leq j \leq J$. For each position $j$ in the final output, we define $g_{j}$ as:

$$
g_{j}=\min _{i} \text { s.t. } o_{i^{\prime}, j^{\prime}}=o_{I, j^{\prime}} \forall i^{\prime} \geq i \text { and } \forall j^{\prime} \leq j
$$

that is, the number of source tokens read before the prefix ending in $j$ took on its final value. The Content Delay row in Table 1 shows delays for our running example. Note that content delay is identical to standard delay for streaming systems, which always have stable prefixes.

With this refined $g$, we can make several latency metrics content-aware, including average proportion (Cho and Esipova, 2016), consecutive wait (Gu et al., 2017), average lagging (Ma et al., 2019a), and differentiable average lagging (Arivazhagan et al., 2019b). We opt for differentiable average lagging (DAL) because of its interpretability and because it sidesteps some problems with average lagging (Cherry and Foster, 2019). It can be thought of as the average number of source tokens a system lags behind a perfectly simultaneous translator:

$$
\mathrm{DAL}=\frac{1}{J} \sum_{j=1}^{J}\left[g_{j}^{\prime}-\frac{j-1}{\gamma}\right]
$$

where $\gamma=J / I$ accounts for the source and target having different lengths, and $g^{\prime}$ adjusts $g$ to incorporate a minimal time cost of $\frac{1}{\gamma}$ for each token:

$$
g_{j}^{\prime}= \begin{cases}g_{j} & j=1 \\ \max \left[g_{j}, g_{j-1}^{\prime}+\frac{1}{\gamma}\right] & j>1\end{cases}
$$




\begin{tabular}{|c|c|c|c|c|c|c|c|c|c|}
\hline Source & Outpu & & & & & & & & Erasure \\
\hline 1: Neue & New & & & & & & & & - \\
\hline 2: Arzneimittel & New & Medicines & & & & & & & 0 \\
\hline 3: könnten & New & Medicines & & & & & & & 0 \\
\hline 4: Lungen- & New & drugs & may & be & lung & & & & 1 \\
\hline 5: und & New & drugs & could & be & lung & and & & & 3 \\
\hline 6: Eierstockkrebs & New & drugs & may & be & lung & and & ovarian & cancer & 4 \\
\hline 7: verlangsamen & New & drugs & may & slow & lung & and & ovarian & cancer & 5 \\
\hline Content Delay & 1 & 4 & 6 & 7 & 7 & 7 & 7 & 7 & \\
\hline
\end{tabular}

Table 1: An example prefix translation list for the tokenized German sentence, "Neue Arzneimittel könnten Lungenund Eierstockkrebs verlangsamen", with reference, "New drugs may slow lung, ovarian cancer".

Note that DAL sums over the final number of target tokens $(J)$, but it is possible for intermediate hypotheses to have more than $J$ tokens. Any such tokens are ignored by DAL.

\subsection{Stability}

Following Niehues et al. $(2016,2018)$ and Arivazhagan et al. (2019a), we measure stability with erasure, which measures the length of the suffix that is deleted to produce the next revision. Let $o_{i}$ be the $i^{t h}$ output of a PTL. The normalized erasure (NE) for PTL is defined as:

$$
\mathrm{NE}=\frac{1}{J} \sum_{i=2}^{I}\left[\left|o_{i-1}\right|-\left|\operatorname{LCP}\left(o_{i}, o_{i-1}\right)\right|\right]
$$

where the $|\cdot|$ operator returns the length of a token sequence, and LCP calculates the longest common prefix of two sequences. Table 1 shows pointwise erasures for each output; its NE would be $13 / 8=$ 1.625 , interpretable as the number of intermediate tokens deleted for each final token.

\section{Re-translation Methods}

To evaluate re-translation, we build up the source sentence one token at a time, translating each resulting source prefix from scratch to construct the PTL for evaluation.

\subsection{Prefix Training}

Standard models trained on full sentences are unlikely to perform well when applied to prefixes. We alleviate this problem by generating prefix pairs from our parallel training corpus, and subsequently training on a 1:1 mix of full-sentence and prefix pairs (Niehues et al., 2018; Dalvi et al., 2018). Following Niehues et al. (2018), we augment our training data with prefix pairs created by selecting a source prefix length uniformly at random, then selecting a target length either proportionally according to sentence length, or based on self-contained word alignments. For the latter, for each source prefix, we attempt to find a target prefix such that all tokens in the source prefix align only to words in the target prefix and vice versa. In preliminary experiments, we confirmed a finding by Niehues et al. (2018) that word-alignment-based prefix selection is no better than proportional selection, so we report results only for the proportional method. ${ }^{1}$ An example of proportional prefix training is given in Table 2. With prefix training, we expect intermediate translations of source prefixes to be shorter, and to look more like partial target prefixes than complete target sentences (Niehues et al., 2018).

\subsection{Inference-time Heuristics}

To improve stability, Arivazhagan et al. (2019a) propose a combination of biased search and delayed predictions. Biased search encourages the system to respect its previous predictions by modifying search to interpolate between the distribution from the NMT model (with weight $1-\beta$ ) and the one-hot distribution formed by the system's translation of the previous prefix (with weight $\beta$ ). We only bias a hypothesis for as long as it strictly follows the previous translation. No bias is applied after the first point of divergence.

To delay predictions until more source context is available, we adopt Ma et al. (2019a)'s wait- $k$ approach at inference time. We implement this by truncating the target to $\max (i-k, 0)$ tokens, where $i$ is the current source prefix length and $k$ is a constant inference-time hyper-parameter. To

\footnotetext{
${ }^{1}$ Word-alignment-based prefix selection may become more important when working on more distant language pairs such as English-Japanese.
} 


\begin{tabular}{|ccl|}
\hline Full & Source & $\begin{array}{l}\text { Die Führungskräfte der Republikaner rechtfertigen ihre Politik mit der } \\
\text { Notwendigkeit, den Wahlbetrug zu bekämpfen [15 tokens] } \\
\text { Target }\end{array}$ \\
& $\begin{array}{l}\text { Republican leaders justified their policy by the need to combat electoral } \\
\text { fraud [12 tokens] }\end{array}$ \\
Prefix & $\begin{array}{c}\text { Source } \\
\text { Target }\end{array}$ & $\begin{array}{l}\text { Die Führungskräfte der Republikaner rechtfertigen [5 tokens] } \\
\text { Republican leaders justified their [4 tokens] }\end{array}$
\end{tabular}

Table 2: An example of proportional prefix training. Each example in the minibatch has a $50 \%$ chance to be truncated, in which case, we truncate its source and target to a randomly-selected fraction of their original lengths, $1 / 3$ in this example. No effort is made to ensure that the two halves of the prefix pair are semantically equivalent.

avoid confusion with Ma et al. (2019a)'s wait- $k$ training, we refer to wait- $k$ used for re-translation as wait-k inference. ${ }^{2}$

\section{Experiments}

We use standard WMT14 English-to-French (EnFr; 36.3M sentences) and WMT15 German-to-English (DeEn; 4.5M sentences) data. For EnFr, we use newstest $2012+2013$ for development, and newstest 2014 for test. For DeEn, we validate on newstest 2013 and report results on newstest 2015. We use BPE (Sennrich et al., 2016) on the training data to construct a $32 \mathrm{~K}$-type vocabulary that is shared between the source and target languages.

\subsection{Models}

Our streaming and re-translation models are implemented in Lingvo (Shen et al., 2019), sharing architecture and hyper-parameters wherever possible. Our RNMT+ architecture (Chen et al., 2018) consists of a 6 layer LSTM encoder and an 8 layer LSTM decoder with additive attention (Bahdanau et al., 2014). Both encoder and decoder LSTMs have 512 hidden units, apply per-gate layer normalization (Ba et al., 2016), and use residual skip connections after the second layer.

The models are regularized using a dropout of 0.2 and label smoothing of 0.1 (Szegedy et al., 2016). Models are optimized using 32-way data parallelism with Google Cloud's TPUv3, using Adam (Kingma and Ba, 2015) with the learning rate schedule described in Chen et al. (2018) and a batch size of 4,096 sentence-pairs. Checkpoints for the base models are selected based on development perplexity.

\footnotetext{
${ }^{2}$ When wait- $k$ truncation is combined with beam search, its behavior is similar to that of Zheng et al. (2019b): sequences are scored accounting for "future" tokens that will not be shown to the user.
}

Streaming We train several wait $k$ training and MILk models to obtain a range of quality-latency trade-offs. Five wait $k$ training models are trained with sub-word level waits of $2,4,6,8$, and 10 . Five MILk models are trained with latency weights of $0.1,0.2,0.3,0.4,0.5$ and 0.75 ; weights lower than 0.1 tend to increase lag without improving BLEU. All streaming models use unidirectional encoders and greedy search.

Re-translation We test two NMT architectures with re-translation: a Base system with unidirectional encoding and greedy search, designed for fair comparisons to our streaming baselines above; and a more powerful Bidi+Beam system using bidirectional encoding and beam search of size 20 , designed to test the impact of an improved base model. Training data is augmented through the proportional prefix training method unless stated otherwise ( $\S 4.1)$. Beam-search bias $\beta$ is varied in the range 0.0 to 1.0 in increments of 0.2. When wait- $k$ inference is enabled, $k$ is varied in $1,2,4$, $6,8,10,15,20,30$. Note that we do not need to re-train to test different values of $\beta$ or $k$.

\subsection{Translation with few revisions}

Biased search and wait- $k$ inference used together can reduce re-translation's revisions, as measured by normalized erasure (NE in $\S 3.3$ ), to negligible levels (Arivazhagan et al., 2019a). But how does retranslation compare to competing approaches? To answer this, we compare the quality-latency tradeoffs achieved by re-translation in a low-revision regime to those of our streaming baselines.

First, we need a clear definition of low-revision re-translation. By manual inspection on the DeEn development set, we observe that systems with an $\mathrm{NE}$ of 0.2 or lower display many different latencyquality trade-offs. But is NE stable across evaluation sets? When we compare development and 

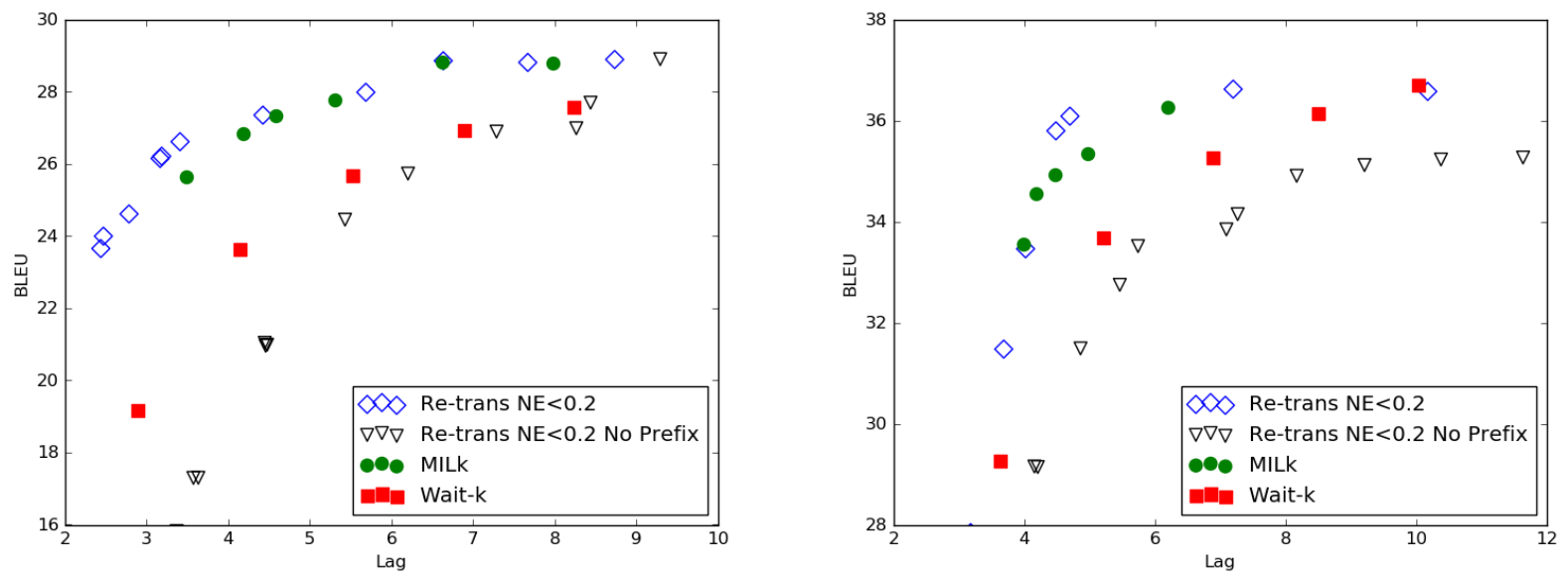

Figure 1: BLEU vs lag (DAL) curves for translation with low erasure for DeEn (left) and EnFr (right) test sets.
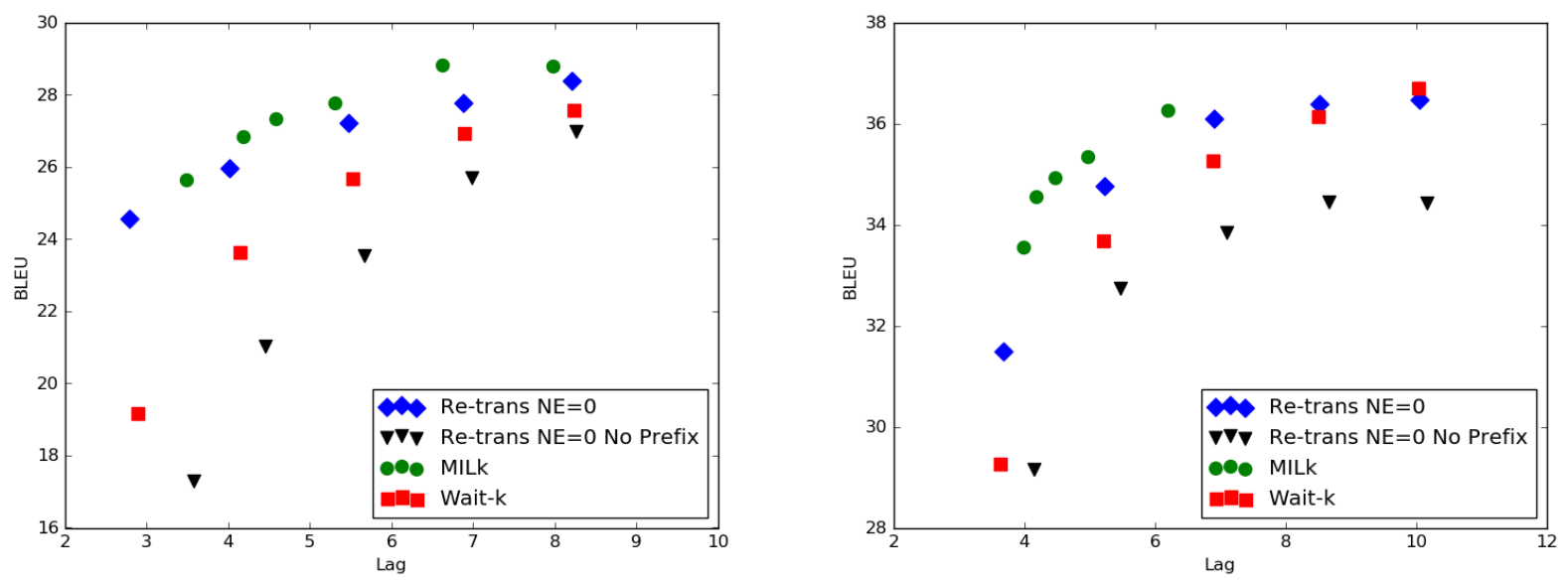

Figure 2: BLEU vs lag (DAL) curves for translation with no erasure for DeEn (left) and EnFr (right) test sets.

test NE for all 50 non-zero-erasure combinations of $\beta$ and $k$, the average absolute difference is 0.005 for DeEn, and 0.004 for EnFr, indicating that development NE is very predictive of test NE. This gives us an operational definition of low-revision re-translation as any configuration with a dev NE $<0.2$, allowing on average less than 1 token to be revised for every 5 tokens in the system output.

Since we need to vary both $\beta$ and $k$ for our retranslation systems, we plot BLEU versus DAL curves by finding the Pareto frontier on the dev set, and then projecting to the test set. To ensure a fair comparison to our baselines, we test only the Base system here. As an ablation, we include a variant that does not use proportional prefixes, and instead trains only on full sentences.

Figure 1 shows our results. Re-translation is nicely separated from wait- $k$, and intertwined with the adaptive MILk. In fact, it is noticeably better than MILk at several latency levels for EnFr. Since re-translation is not adaptive, this indicates that being able to make a small number of revisions is quite advantageous for finding good quality-latency trade-offs. On the other hand, the ablation curve, "Re-trans NE $<0.2$ No Prefix" is much worse, indicating that proportional prefix training is very valuable in this setting. We probe its value further in the next experiment.

\subsection{Translation with no revisions}

Motivated by the strong performance of retranslation with few revisions, we now evaluate it with no revisions, by setting $\beta$ to 1 , which guarantees NE $=0$. Since $\beta$ is locked at 1 , we can build a curve by varying $k$ from 2 to 10 in increments of 2. In this setting, re-translation becomes equivalent to wait- $k$ inference without wait- $k$ training, which is studied as an ablation to wait- $k$ training by Ma et al. (2019a). ${ }^{3}$ However, where they tested

\footnotetext{
${ }^{3}$ Re-translation with beam search and with $\beta=1$ is similar to wait- $k$ inference with speculative beam search (Zheng et al., 2019 b), due to effective look-ahead from implementing wait- $k$
} 

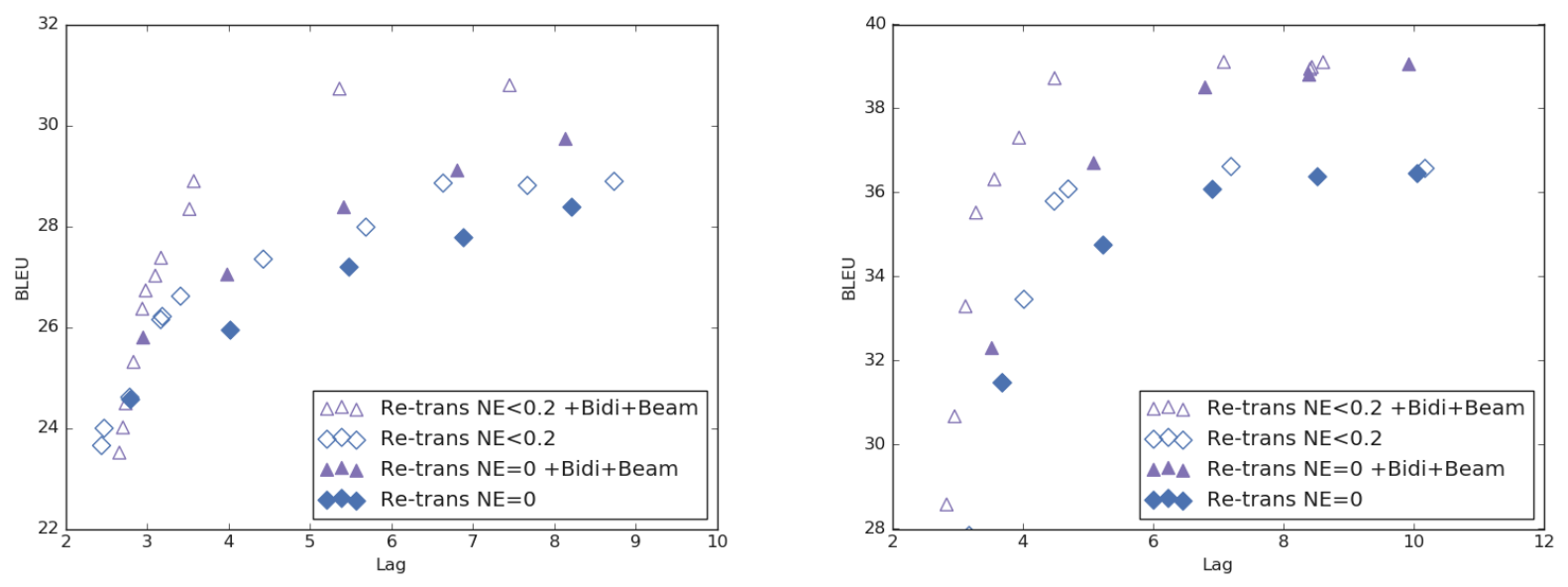

Figure 3: BLEU vs lag (DAL) curves for re-translation with improved models for DeEn (left) and EnFr (right) test sets.

wait- $k$ inference on a system with full-sentence training, we do so for a system with proportional prefix training ( $\S 4.1$ ). As before, we compare to our streaming baselines, test only our Base system, and include a no-prefix ablation corresponding to full-sentence training.

Results are shown in Figure 2. First, retranslation outperforms wait- $k$ training at almost all latency levels. This is startling, because each wait- $k$ training point is trained specifically for its $k$, while the re-translation points reflect a single training run, reconfigured for different latencies by adjusting $k$ at test time. We suspect that this improvement stems from prefix-training introducing stochasticity to the amount of source context used to predict target words, making the model more robust. Second, without prefix training, re-translation is consistently below wait- $k$ training, confirming earlier experiments by Ma et al. (2019a) on the ineffectiveness of wait- $k$ inference without specialized training, and confirming our earlier observations on the surprising effectiveness of prefix training. Finally, we see that even without revisions, re-translation is very close to MILk, suggesting that this combination of prefix training and wait- $k$ inference is an extremely strong baseline, even for a 0 -revision regime.

\subsection{Extendability of re-translation}

Re-translation's primary strengths lie in its ability to revise and its ability to apply to any MT system. With some effort, streaming systems can be fitted with enhancements such as bidirectional en-

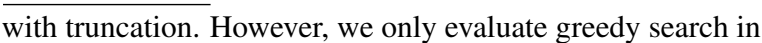
this comparison, where their equivalence is exact. coding (Ma et al., 2019a), ${ }^{4}$ beam search (Zheng et al., 2019b) and multihead attention (Ma et al., 2019b). Conversely, re-translation can wrap any auto-regressive NMT system and immediately benefit from its improvements. Furthermore, retranslation's latency-quality trade-off can be manipulated without retraining the base system. It is not the only solution to have these properties; most policies that are not trained jointly with NMT can make the same claims (Cho and Esipova, 2016; Gu et al., 2017; Zheng et al., 2019a). We conduct an experiment to demonstrate the value of this flexibility, by comparing our Base system to the upgraded Bidi+Beam. ${ }^{5}$ We carry out this test with few revisions $(\mathrm{NE}<0.2)$ and without revisions $(\mathrm{NE}=0)$, projecting Pareto curves from dev to test where necessary. The results are shown in Figure 3.

Comparing the few-revision ( $\mathrm{NE}<0.2)$ curves, we see large improvements, some more than 2 BLEU points, from using better models. Looking at the no-revision $(\mathrm{NE}=0)$ curves, we see that this configuration also benefits from modeling improvements, but for DeEn, the deltas are noticeably smaller than those of the few-revision curves.

\subsection{On computational complexity}

Re-translation is conceptually simple and easy to implement, but also incurs an increase in asymptotic time complexity. If the base model can translate a sentence in time $O(x)$, then re-translation

\footnotetext{
${ }^{4}$ Any streaming model with a bidirectional encoder requires re-encoding for each source prefix, resulting in higher compute and memory costs.

${ }^{5} \mathrm{We}$ could just as easily upgrade to a different base architecture, such as the Transformer (Vaswani et al., 2017), which could potentially to lead to further improvements.
} 
takes $O(n x)$ where $n$ is the number of times we request re-translation for that sentence. $n$ is capped at the length of the sentence, as we never revise translations of earlier sentences in the transcript. ${ }^{6}$

For many settings, this increase in complexity can be easily ignored. We are not concerned with the total time to translate a sentence, but instead with the latency between a new source word being uttered and its translation's appearance on the screen. Modern accelerators can translate a complete sentence in the range of 100 milliseconds, ${ }^{7}$ meaning that the time required to update the screen by translating an updated source prefix is small enough to be imperceptible. As in all simultaneous systems, the largest source of latency is waiting for new source content to arrive. ${ }^{8}$

\section{Conclusion}

We have presented the first comparison of retranslation and streaming strategies for simultaneous translation. We have shown re-translation with low levels of erasure $(\mathrm{NE}<0.2)$ to be as good or better than the state of the art in streaming translation. Also, re-translation easily embraces arbitrary improvements to NMT, which we have highlighted with large gains from an upgraded base model.

In our setting, re-translation with no erasure reduces to wait- $k$ inference, which we have shown to be much more effective than previously reported, so long as the underlying NMT system's training data has been augmented with prefix pairs. Due to its simplicity and its effectiveness, we suggest retranslation as a strong baseline for future research on simultaneous translation.

\section{References}

Naveen Arivazhagan, Colin Cherry, Te I, Wolfgang Macherey, Pallavi Baljekar, and George Foster.

\footnotetext{
${ }^{6}$ Though our experiments in this paper are sentence-level for the sake of continuity with earlier work on streaming translation, in practice, source sentence boundaries can be provided by speech-recognition endpointing or unspoken punctuation prediction (Arivazhagan et al., 2019a).

${ }^{7} 24.43$ milliseconds per sentence is achievable on a NVIDIA TITAN RTX, according to MLPerf v0.5 Inference Closed NMT Stream. Retrieved from www.mlperf.org November 6th, 2019, entry Inf-0.5-27. MLPerf name and logo are trademarks. See www.mlperf.org for more information.

${ }^{8}$ We acknowledge the desirability of low computational complexity, both for reducing the cumulative burden alongside other sources of latency, such as network delays, and for reducing power consumption for mobile or green computing. Finding a lower-cost solution that preserves the flexibility of re-translation is a worthy goal for future research.
}

2019a. Re-translation strategies for long form, simultaneous, spoken language translation. CoRR, abs/1912.03393.

Naveen Arivazhagan, Colin Cherry, Wolfgang Macherey, Chung-Cheng Chiu, Semih Yavuz, Ruoming Pang, Wei Li, and Colin Raffel. 2019b. Monotonic infinite lookback attention for simultaneous machine translation. In Proceedings of the 57th Annual Meeting of the Association for Computational Linguistics, pages 1313-1323, Florence, Italy. Association for Computational Linguistics.

Jimmy Lei Ba, Jamie Ryan Kiros, and Geoffrey E. Hinton. 2016. Layer Normalization. arXiv e-prints, page arXiv:1607.06450.

Dzmitry Bahdanau, Kyunghyun Cho, and Yoshua Bengio. 2014. Neural machine translation by jointly learning to align and translate. arXiv preprint arXiv:1409.0473.

Mia Xu Chen, Orhan Firat, Ankur Bapna, Melvin Johnson, Wolfgang Macherey, George Foster, Llion Jones, Mike Schuster, Noam Shazeer, Niki Parmar, Ashish Vaswani, Jakob Uszkoreit, Lukasz Kaiser, Zhifeng Chen, Yonghui Wu, and Macduff Hughes. 2018. The best of both worlds: Combining recent advances in neural machine translation. In Proceedings of the 56th Annual Meeting of the Association for Computational Linguistics (Volume 1: Long Papers), pages 76-86. Association for Computational Linguistics.

Colin Cherry and George Foster. 2019. Thinking slow about latency evaluation for simultaneous machine translation. CoRR, abs/1906.00048.

Kyunghyun Cho and Masha Esipova. 2016. Can neural machine translation do simultaneous translation? CoRR, abs/1606.02012.

Fahim Dalvi, Nadir Durrani, Hassan Sajjad, and Stephan Vogel. 2018. Incremental decoding and training methods for simultaneous translation in neural machine translation. In Proceedings of the 2018 Conference of the North American Chapter of the Association for Computational Linguistics: Human Language Technologies, Volume 2 (Short Papers), pages 493-499. Association for Computational Linguistics.

Jiatao Gu, Graham Neubig, Kyunghyun Cho, and Victor O.K. Li. 2017. Learning to translate in real-time with neural machine translation. In Proceedings of the 15th Conference of the European Chapter of the Association for Computational Linguistics: Volume 1, Long Papers, pages 1053-1062. Association for Computational Linguistics.

Diederik P. Kingma and Jimmy Ba. 2015. Adam: A method for stochastic optimization. In International Conference on Learning Representations. 
Mingbo Ma, Liang Huang, Hao Xiong, Renjie Zheng, Kaibo Liu, Baigong Zheng, Chuanqiang Zhang, Zhongjun He, Hairong Liu, Xing Li, Hua Wu, and Haifeng Wang. 2019a. STACL: Simultaneous translation with implicit anticipation and controllable latency using prefix-to-prefix framework. In Proceedings of the 57th Annual Meeting of the Association for Computational Linguistics, pages 3025-3036, Florence, Italy. Association for Computational Linguistics.

Xutai Ma, Juan Pino, James Cross, Liezl Puzon, and Jiatao Gu. 2019b. Monotonic multihead attention. CoRR, abs/1909.12406.

Jan Niehues, Thai Son Nguyen, Eunah Cho, Thanh-Le Ha, Kevin Kilgour, Markus Müller, Matthias Sperber, Sebastian Stüker, and Alex Waibel. 2016. Dynamic transcription for low-latency speech translation. In Interspeech, pages 2513-2517.

Jan Niehues, Ngoc-Quan Pham, Thanh-Le Ha, Matthias Sperber, and Alex Waibel. 2018. Lowlatency neural speech translation. In Proc. Interspeech 2018, pages 1293-1297.

Kishore Papineni, Salim Roukos, Todd Ward, and WeiJing Zhu. 2002. Bleu: a method for automatic evaluation of machine translation. In Proceedings of the 40th Annual Meeting of the Association for Computational Linguistics.

Rico Sennrich, Barry Haddow, and Alexandra Birch. 2016. Neural machine translation of rare words with subword units. In Proceedings of the 54th Annual Meeting of the Association for Computational Linguistics (Volume 1: Long Papers), pages 1715-1725. Association for Computational Linguistics.

Jonathan Shen, Patrick Nguyen, Yonghui Wu, et al. 2019. Lingvo: a modular and scalable framework for sequence-to-sequence modeling. CoRR, abs/1902.08295.

Christian Szegedy, Vincent Vanhoucke, Sergey Ioffe, Jonathon Shlens, and Zbigniew Wojna. 2016. Rethinking the inception architecture for computer vision. 2016 IEEE Conference on Computer Vision and Pattern Recognition (CVPR), pages 2818-2826.

Ashish Vaswani, Noam Shazeer, Niki Parmar, Jakob Uszkoreit, Llion Jones, Aidan N Gomez, Łukasz Kaiser, and Illia Polosukhin. 2017. Attention is all you need. In Advances in neural information processing systems, pages 5998-6008.

Baigong Zheng, Renjie Zheng, Mingbo Ma, and Liang Huang. 2019a. Simpler and faster learning of adaptive policies for simultaneous translation. In Proceedings of the 2019 Conference on Empirical Methods in Natural Language Processing and the 9th International Joint Conference on Natural Language Processing (EMNLP-IJCNLP), pages 1349_ 1354, Hong Kong, China. Association for Computational Linguistics.
Renjie Zheng, Mingbo Ma, Baigong Zheng, and Liang Huang. 2019b. Speculative beam search for simultaneous translation. In Proceedings of the 2019 Conference on Empirical Methods in Natural Language Processing and the 9th International Joint Conference on Natural Language Processing (EMNLPIJCNLP), pages 1395-1402, Hong Kong, China. Association for Computational Linguistics. 